\title{
Quantum-Mechanical Investigation of Field-Emission Mechanism of a Micrometer-Long Single-Walled Carbon Nanotube
}

\author{
Xiao Zheng, ${ }^{1}$ GuanHua Chen, ${ }^{1,2, *}$ Zhibing Li, ${ }^{2}$ Shaozhi Deng, ${ }^{2}$ and Ningsheng $\mathrm{Xu}^{2, \dagger}$ \\ ${ }^{1}$ Department of Chemistry, The University of Hong Kong, Hong Kong, China \\ ${ }^{2}$ Department of Physics, Zhongshan University, Guangzhou, China
}

(Received 24 October 2003; published 12 March 2004)

\begin{abstract}
A quantum-mechanical simulation is carried out to investigate the charge distribution and electrostatic potential along a $1 \mu \mathrm{m}$ long $(5,5)$ single-walled carbon nanotube under realistic field-emission experimental conditions. A single layer of carbon atoms is found sufficient to shield most of the electric field except at the tip where strong field penetration occurs. The penetration leads to a nonlinear decrease of potential barrier for emission, which is equally responsible for the low threshold voltage besides the well-known geometrical field enhancement factor.
\end{abstract}

DOI: 10.1103/PhysRevLett.92.106803

PACS numbers: 73.22.-f, 73.21.-b, 73.63.Fg, 79.70.+q

Very high aspect ratios lead to extremely strong local fields and high current densities for carbon nanotubes (CNTs) as electron field emitters [1]. Under a strong applied external field, it was found that the classical FowlerNordheim law [2] is no longer valid [3,4]. Simulations based on density-functional theory have been performed to understand the field-emission mechanism [5]. Limited by the computational efficiencies and resources, only the CNT tips of a few $\mathrm{nm}$ in length were considered explicitly. This was justified under the assumption that the external fields can be scaled by $\left(L_{\text {tube }} / L_{\text {tip }}\right)$ times for the shortened CNTs to produce the same electrostatic fields around the tips [6], where $L_{\text {tip }}$ and $L_{\text {tube }}$ are the lengths of the tip used in the calculation and the actual tube, respectively. The scaled field $E_{\text {scal }}$ is much stronger than the applied field $E_{\text {appl }}$, which leads to the localized electronic states at the very tip where the electrons are expected to emit [7]. The validity of this assumption needs to be examined. Further, the amount of extra charges around the tip had not been determined and was varied to assess their effects in previous calculations [5]. Classically a metallic field emitter has the same electrostatic potential as the cathode, and the electrostatic field is completely shielded by the charges on the surface of the emitter. However, CNTs have very thin tube walls. It is known that field penetration lengths for metals are a few angstroms or larger [8], and it is thus interesting to examine whether the electric field penetrates the tube, which may result in an unconventional field-emission mechanism. When there are excess charges distributing along the tube, we must consider the entire tube and the substrate since the Coulomb interaction is long ranged. All these call for the explicit treatment of the entire tube and boundary condition in the simulation. In this work we calculate explicitly the charge distributions and electrostatic fields along a $1 \mu \mathrm{m}$ long capped $(5,5)$ CNT under

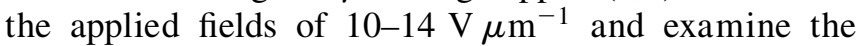
field-emission mechanism.
It is an enormous computational challenge to simulate explicitly a $1 \mu \mathrm{m}$ long $(5,5)$ single-walled carbon nanotube (SWNT) which contains about $10^{5}$ atoms. Figure 1(a) depicts a typical field-emission system. In contrast to the classical field-emission model where the CNT is treated as a bulk metal having the same electrostatic potential as the cathode, the SWNT in our model is taken as a quantum object subjected to the following boundary condition as depicted in Fig. 1(a):

$$
V= \begin{cases}V_{a}>0 & \text { anode } \\ 0 & \text { cathode. }\end{cases}
$$

To simulate the entire tube, we adopt a hybrid quantum mechanics and molecular mechanics (QM/MM) approach [9]. Limited by computational resources, $\sim 8000$ atoms at or near the tip are simulated quantum mechanically, while the rest are treated as point charges since their predominant influence on the emission is through their electrostatic potential at the tip. Under the applied external field $E_{\text {appl }}$, the energies of local orbitals are lowered. Some of them are lowered below the Fermi energy, and the electrons are induced from the cathode to occupy subsequently these local orbitals. The number of these local orbitals is proportional to their energy
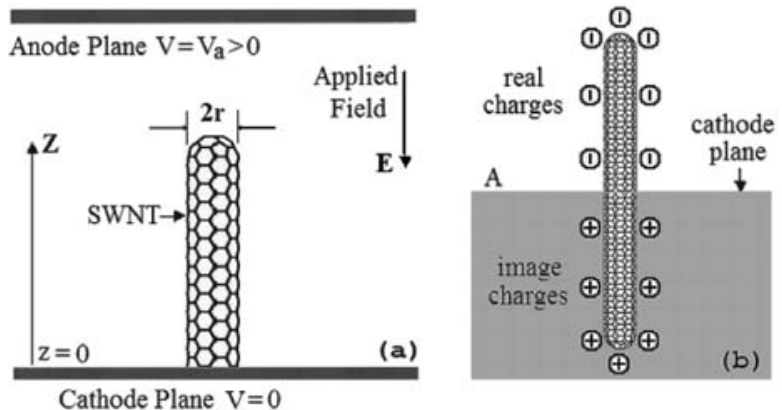

FIG. 1. (a) Field emission setup for a SWNT. (b) Mirror images of the induced charges. 
change, provided that the density of states (DOS) is a constant. Numerical calculations [10] and experiments [11] revealed that the DOS near the Fermi energy is approximately constant. The energy changes of the local orbitals at a position $z$ are approximately the change of local electrostatic potential energy $\delta V(z)$. Therefore, the number of local orbitals whose energies are lowered below the Fermi energy is proportional to $\delta V(z)$; i.e., the induced charge density $n(z) \propto \delta V(z)$. This expression is consistent with the Thomas-Fermi theory and is a good approximation when $n(z)$ or $\delta V(z)$ is relatively small. According to the Poisson equation $\nabla^{2} \delta V(z) \propto n(z)$, we obtain that $\nabla^{2} n(z) \propto n(z)$, and subsequently the linear induced charge density (or charge per unit length) along the tube, $\rho(z)$, can be expressed as

$$
\rho(z) \sim \rho\left(L^{\prime}\right) \exp \left\{-\left(L^{\prime}-z\right) / \lambda\right\},
$$

where $\lambda$ represents the decay length, and $\rho\left(L^{\prime}\right)$ is the linear induced charge density at $z=L^{\prime}$ where QM and MM regions intersect. $\rho\left(L^{\prime}\right)$ can be determined from the quantum-mechanical part of our calculation. We employ the linear-scaling divide-and-conquer (DAC) method [12] to calculate the charge distribution among the $\sim 8000$ atoms in the tip region. Being connected to the cathode, the SWNT can be treated as in quasiequilibrium with the cathode before the electrons emit or when the current is minute. This provides a perfect solution to determine the amount of electrons that the cathode provides to the tube, since the Fermi energy has to be a constant throughout the joint system of the cathode and the SWNT. The boundary condition is further satisfied by introducing the mirror images of the charges distributed along the tube, as illustrated in Fig. 1(b). Tungsten, gold, and carbon fibers have been used frequently as support materials for CNT field emitters. We choose tungsten as the cathode in our simulation. The work functions of tungsten and long neutral $(5,5) \mathrm{CNT}$ are 4.5 and $4.68 \mathrm{eV}$ [13], respectively. The Fermi energy of the joint system is thus $-4.5 \mathrm{eV}$ since the cathode and CNT are in quasiequilibrium. The applied external field ranges from 10 to

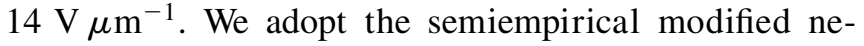
glect of diatomic overlap model [14] in the DAC calculation. The QM region is divided into subunits which are simulated one at a time. Buffer zones are introduced at the boundaries of neighboring subunits to account for the chemical bonds and near-field interactions. Hydrogen atoms are further introduced to terminate the dangling bonds. There are about 120 to 400 atoms within every combined subunit/buffer zones. The values of $\lambda$ and $\rho\left(L^{\prime}\right)$ are updated at each iteration of the DAC calculation until the induced charge distribution $\rho(z)$ along the entire tube converges. Usually three or more iterations are needed to achieve the convergence.

In Fig. 2 we plot the induced charge distributions at the cap and along the tube for the external field $14 \mathrm{~V} \mu \mathrm{m}^{-1}$. Figure 2(a) depicts the induced electron

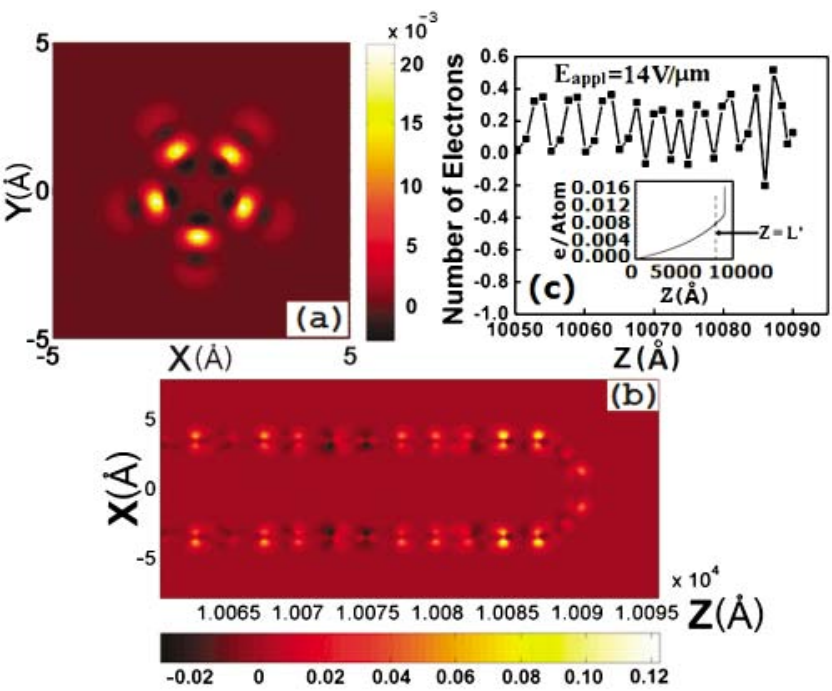

FIG. 2 (color). (a) Induced charge distribution at the top layer of the SWNT's cap. (b) Induced charge distribution in a middle plane bisecting the tube. (c) Number of induced electrons on each layer along the $z$ axis in the tip region. Excessive charges on the entire tube are depicted in the inset. All charge densities in (a) and (b) are in units of electron $/ \AA^{3}$.

density in a plane that goes through the five atoms at the end of the cap, and Fig. 2(b) is the induced charge distribution in a middle plane bisecting the tube. We find that the induced charges are only a few percentages of the total number of electrons at the tip. Figure 2(c) shows the induced charges layer by layer along the $z$ axis. A standing wave is observed for the induced charges. The inset of Fig. 2(c) depicts the excess charges for the entire tube, which are found concentrating at the tip region (similar to the classical metallic tube). $\lambda$ is $0.48 \mu \mathrm{m}$ and $\rho\left(L^{\prime}\right) \quad 0.66$ electron $/ \mathrm{nm}$ for $E_{\text {appl }}=14 \mathrm{~V} \mu \mathrm{m}^{-1}$, while $\lambda$ is $0.45 \mu \mathrm{m}$ and $\rho\left(L^{\prime}\right) 0.50$ electron $/ \mathrm{nm}$ for

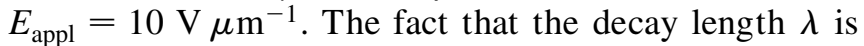
almost half a micrometer confirms the necessity to consider explicitly the entire carbon nanotube. We vary the number of atoms in the $\mathrm{QM}$ region to examine their influence on the outcome. The induced charge distribution changes little with the number varying between 4000 and 8000 and fluctuates drastically as the number varies between 1000 and 2000. This reflects the fact that the induced charges decay rapidly among the first $\sim 1000$ atoms at the tip, as indicated in Fig. 2(c).

In Fig. 3 we plot the potential energy contours for an electron under the external field of $14 \mathrm{~V} \mathrm{~m}^{-1}$. It is significant to note that the potential drop concentrates mostly at the tip region; see Fig. 3(a). This indicates that the electric field penetrates strongly at the tip. However, the variation of electrostatic potential along most of the tube is only about $2 \mathrm{~V}$, much less than the applied bias voltage. Figure $3(\mathrm{~b})$ is the amplified potential contour plot around the cap. The deep blue region is the tube wall, and the dotted line is the energy contour for the 


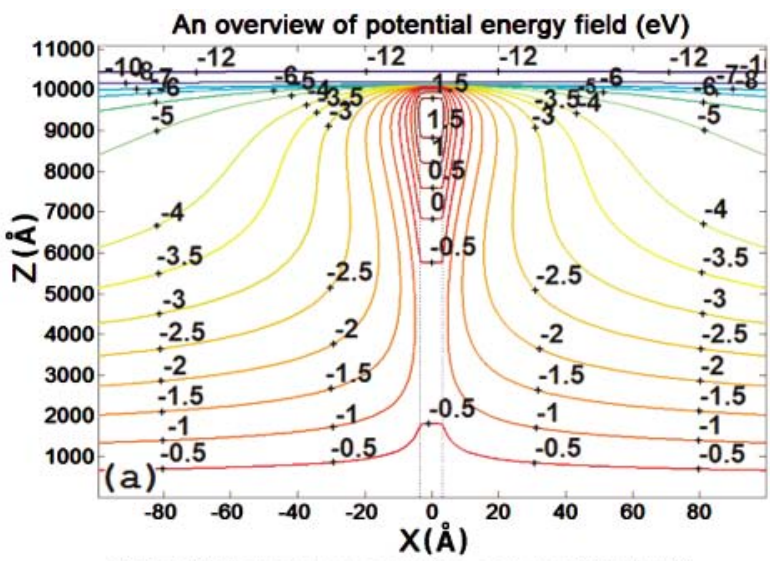

Potential energy near the cap region (eV)

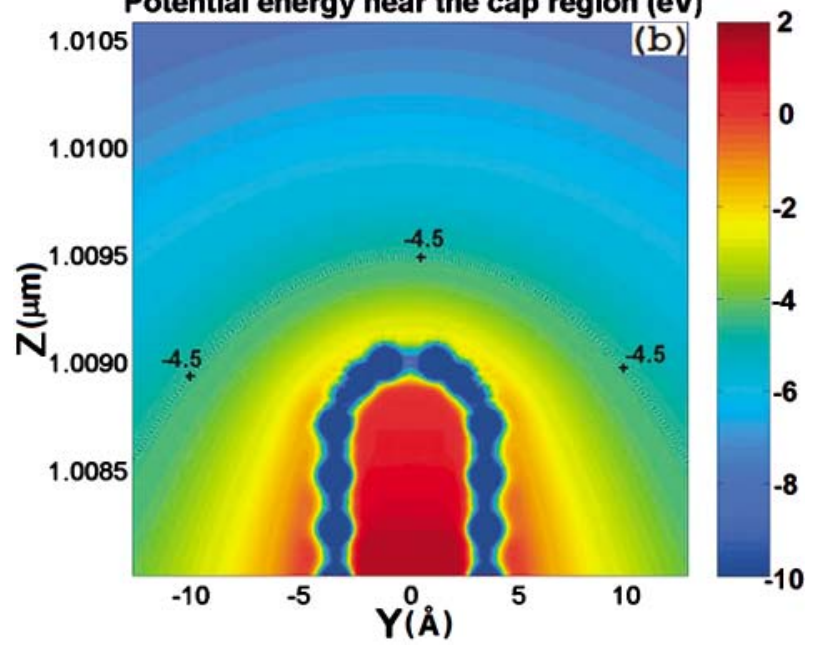

FIG. 3 (color). Potential energy contour plots (a) for the $(5,5)$ SWNT and (b) in the vicinity of the cap under $E_{\text {appl }}=$ $14 \mathrm{~V} \mu \mathrm{m}^{-1}$. To keep the image clear, the core potential is cut at $-10 \mathrm{eV}$. The dotted line in (b) is the equipotential line for the Fermi energy $(-4.5 \mathrm{eV})$.

Fermi energy $(-4.5 \mathrm{eV})$. Clearly the Fermi energy is below the potential barriers around the tip. The electrostatic field is much stronger outside the tube as compared to that inside. Potential barriers on the sidewall are much higher and thicker than that in front of the cap. Forward emission from the cap has the minimum barrier height and thickness and is thus expected. Similar potential contour plot is obtained for $E_{\text {appl }}=10 \mathrm{~V} \mathrm{~mm}^{-1}$ with weaker field penetration. Figure 4(a) shows the electrostatic potentials along the central axis at the tip for three

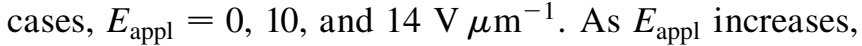
the barrier height is lowered. In Table I, we list the values of barrier height. Obviously, the barrier height depends nonlinearly on the external field and is lowered by as much as $2.5 \mathrm{eV}$ for $E_{\text {appl }}=14 \mathrm{~V} \mathrm{\mu m}^{-1}$. Meanwhile, its thickness decreases rapidly and the emission current intensifies drastically. We emphasize that the lowering of the potential barrier is not caused by the image charges of the emitting electrons [15]. The local fields in front of the tip averaged over the range from $z=10091.5$ to $10095 \AA$

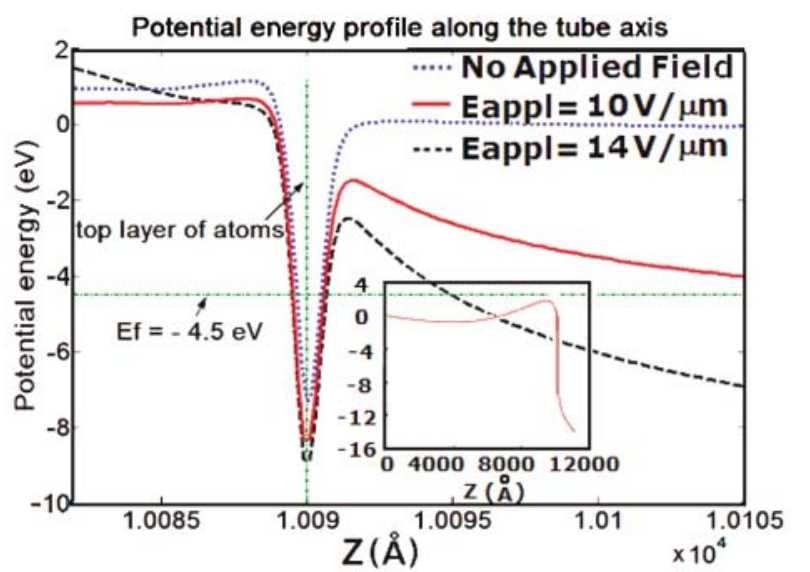

Electrostatic potential along the A-B-C-D path

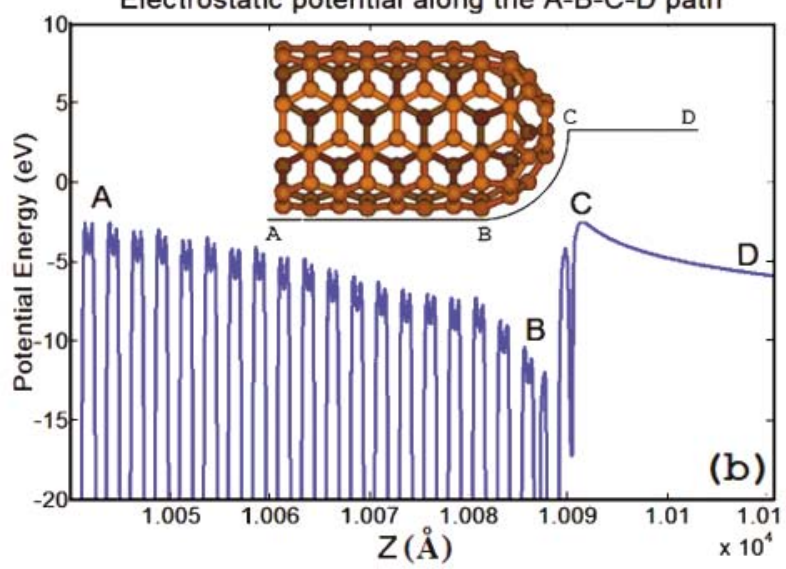

FIG. 4 (color). (a) Potential energy profiles in the vicinity of the cap under different applied fields; data are extracted along the central axis of the carbon nanotube. The electrostatic potential along the entire tube for $E_{\text {appl }}=10 \mathrm{~V} \mathrm{\mu m}^{-1}$ is presented in the inset. (b) Electrostatic potential along the $A-B-C-D$ path which is embedded in the tube wall as specified

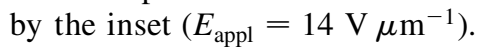

are 3.1 and $5.7 \mathrm{Vnm}^{-1}$ for $E_{\text {appl }}=10$ and $14 \mathrm{~V} \mathrm{~m}^{-1}$, respectively. The corresponding ratios of the local field to the applied field are thus 310 and 410, respectively. The lowering of the barrier height due to the field penetration increases further the effective field enhancement factors which are found 500 and 1200 for $E_{\text {appl }}=10$ and $14 \mathrm{~V} \mu \mathrm{m}^{-1}$, respectively. Figure $4(\mathrm{~b})$ is the electrostatic potential plotted along the $A-B-C-D$ path. A steep potential well is observed between $z=10040$ and $10090 \AA$, which is similar to the bending of conduction bands of semiconductors under the applied field. This phenomenon is distinctively different from that of the conventional

TABLE I. The potential barrier height $\phi$ with respect to the Fermi level under the applied field $E_{\text {appl }}=0,10$, and $14 \mathrm{~V} \mu \mathrm{m}^{-1}$.

\begin{tabular}{cccc}
\hline \hline$E_{\text {appl }}$ & 0 & $E_{\text {appl }}=10{\mathrm{~V} \mu \mathrm{m}^{-1}}$ & $E_{\text {appl }}=14 \mathrm{~V} \mathrm{\mu m}^{-1}$ \\
\hline$\phi$ & $4.5 \mathrm{eV}$ & $3.0 \mathrm{eV}$ & $2.0 \mathrm{eV}$ \\
\hline \hline
\end{tabular}


model which treats the emissive carbon nanotubes as ideal metals [16]. Consequently, induced electrons have high density in the tip region with the $0.095(0.13)$ elec-

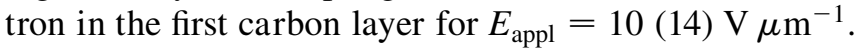
The inset of Fig. 4(a) is the electrostatic potential along the entire tube for $E_{\text {appl }}=10 \mathrm{~V} \mu \mathrm{m}^{-1}$. Similar to that of $E_{\text {appl }}=14 \mathrm{~V} \mathrm{~m}^{-1}$ [cf. Fig. 3(a)], most of the tube is approximately of the same potential. This implies that a single layer of carbon atoms is sufficient to screen the

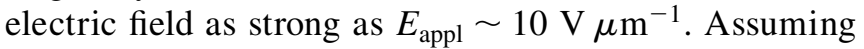
that emitting electrons mainly come from the excess charges in the $\pi^{*}$ states near the Fermi energy, the emission current can be estimated by $J=e \nu q_{\mathrm{exc}} D\left(E_{f}\right)$, where $q_{\text {exc }}$ is the number of excess electrons in the first layer of CNT tip, $\nu$ is the impinging frequency estimated from the $\pi^{*}$ orbital energy $E\left(\pi^{*}\right)$, and $D(E)$ is the transmission probability through the potential barrier estimated with WKB approximation. The emission currents are $0.44 \mathrm{pA}$ $(8.41 \mu \mathrm{A})$ for $E_{\mathrm{appl}}=10(14) \mathrm{V} \mu \mathrm{m}^{-1}$, which are comparable to the experimental findings [15].

Zettl and co-workers performed an electron holography measurement of the electric field distribution along a field-emitting multiwalled CNT [15]. They have found that (i) the field-emission current concentrates precisely at the tip and not at the tube defects such as sidewall imperfections and (ii) the measured holography is consistent with the scenario that there is no potential drop along the micrometer-long tube. Our results are consistent with their findings. This is because $\pi^{*}$ orbitals are empty before the application of electric field and can accommodate enough electrons to shield the electric field. The shielding of the electric field is a natural consequence of the equilibrium condition that the Fermi energy is constant throughout the joint system. While the external field lowers the energies of local orbitals, the electrostatic interactions from the induced electrons bring up the highest occupied molecular orbitals to the Fermi energy of the cathode. As a result, the electric field is shielded for most of the tube while penetrating the tip.

To conclude, we have carried out a detailed quantummechanical simulation of a micrometer-long SWNT under realistic field-emission experimental conditions. Except for the tip, a single layer of carbon atoms is sufficient to shield most of the strong electric field for the bulk of the SWNT. The field penetration at the tip lowers the potential barrier and leads to the deep potential well in the tip region where a large amount of excess electrons reside. The lowering of barrier height leads to a significant increase of emitting current, which reveals the unconventional nature of the CNT field-emission mechanism. The potential barrier height is a nonlinear function of the applied field and is system dependent. It is thus likely that the modification of the tip may lead to a drastic alternation of emission current or threshold voltage.

We thank Dr. XiuJun Wang, Dr. Yang Zhao, Dr. Huasheng Wu, and Dr. S. D. Liang for valuable discussions. X. Z. and G. C. gratefully acknowledge support from the Hong Kong Research Grant Council (RGC). N.X., S. D., and Z. L. gratefully acknowledge financial support of the projects from the National Natural Science Foundation of China (the Distinguished Creative Group Project; Grant No. 90103028), from the Education Ministry of China, from the Higher Education Bureau, and from the Science and Technology Commission of the Guangdong Province.

*Electronic address: ghc@everest.hku.hk

${ }^{\dagger}$ Electronic address: stsxns@zsu.edu.cn

[1] R. H. Baughman, A. A. Zakhidov, and W. A. de Heer, Science 297, 787 (2002); J.-M. Bonard, K. A. Dean, B. F. Coll, and C. Klinke, Phys. Rev. Lett. 89, 197602 (2002).

[2] J.W. Gadzuk and E.W. Plummer, Rev. Mod. Phys. 45, 487 (1973).

[3] R. B. Sharma et al., Chem. Phys. Lett. 344, 283 (2001).

[4] P. G. Collins and A. Zettl, Phys. Rev. B 55, 9391 (1997).

[5] J. Luo, L.-M. Peng, Z. Q. Xue, and J. L. Wu, Phys. Rev. B 66, 155407 (2002).

[6] S. Han, M. H. Lee, and J. Ihm, Phys. Rev. B 65, 085405 (2002).

[7] G. Zhou, W. H. Duan, and B. L. Gu, Phys. Rev. Lett. 87, 095504 (2001).

[8] N. D. Lang and W. Kohn, Phys. Rev. B 1, 4555 (1970).

[9] A. Warshel and M. Levitt, J. Mol. Biol. 103, 227 (1976).

[10] A. M. Rao et al., Science 275, 187 (1997); X. Blase et al., Phys. Rev. Lett. 72, 1878 (1994); R. A. Jishi, J. Bragin, and L. Lou, Phys. Rev. B 59, 9862 (1999).

[11] J.W. G. Wildöer et al., Nature (London) 391, 59 (1998).

[12] W. Yang, Phys. Rev. Lett. 66, 1438 (1991).

[13] J. Zhao, J. Han, and J. P. Lu, Phys. Rev. B 65, 193401 (2002).

[14] M. J. S. Dewar and W. Thiel, J. Am. Chem. Soc. 99, 4899 (1977).

[15] J. Cumings, A. Zettl, M. R. McCartney, and J.C.H. Spence, Phys. Rev. Lett. 88, 056804 (2002).

[16] A. Modinos, Field, Thermionic, and Secondary Electron Emission Spectroscopy (Plenum Publishing Co., New York, 1984) 